OPEN ACCESS

Edited by:

Lucio Vera-Cabrera,

Universidad Autonoma de Nuevo

León, Mexico

Reviewed by:

Jin Wang,

Shenzhen Second People's Hospital,

China

Jorge Castro-Garza,

Instituto Mexicano del Seguro Social,

Mexico

*Correspondence:

Zhenjun $L$

lizhenjun@icdc.cn

${ }^{\dagger}$ These authors have contributed equally to this work

Specialty section:

This article was submitted to Clinical Microbiology,

a section of the journal

Frontiers in Cellular and

Infection Microbiology

Received: 14 December 2021

Accepted: 15 February 2022

Published: 02 March 2022

Citation:

Liu X, Qiu X, Xu S, Che Y, Han L, Kang $Y$, Yue $Y$, Chen S, Li F and Li Z (2022) A CRISPR-Cas12a-Assisted

Fluorescence Platform for Rapid and Accurate Detection of

Nocardia cyriacigeorgica.

Front. Cell. Infect. Microbiol. 12:835213.

do: 10.3389/fcimb.2022.835213

\section{A CRISPR-Cas12a-Assisted Fluorescence Platform for Rapid and Accurate Detection of Nocardia cyriacigeorgica}

\author{
Xueping Liu ${ }^{1+}$, Xiaotong Qiu ${ }^{2+}$, Shuai $\mathrm{Xu}^{2+}$, Yanlin Che ${ }^{1}$, Lichao Han ${ }^{2}$, Yutong Kang ${ }^{2}$, \\ Yuan Yue ${ }^{3}$, Shenglin Chen ${ }^{4}$, Fang $\mathrm{Li}^{5}$ and Zhenjun $\mathrm{Li}^{1,2^{*}}$ \\ ${ }^{1}$ School of Laboratory Medicine and Life Sciences, Wenzhou Medical University, Wenzhou, China, ${ }^{2}$ State Key Laboratory of \\ Infectious Disease Prevention and Control, National Institute for Communicable Disease Control and Prevention, Chinese \\ Center for Disease Control and Prevention, Beiijing. China, ${ }^{3}$ Key Laboratory of the Ministry of Education for the Conservation \\ and Utilization of Special Biological Resources of Western China, Ningxia University, Yinchuan, China, ${ }^{4}$ School of Public \\ Health, Shanxi Medical University, Taiyuan, China, ${ }^{5}$ Department of Medicine, Tibet University, Lhasa, China
}

Nocardia cyriacigeorgica has gradually become a common pathogen in clinical microbial infections. Identification of Nocardia at the species level is essential to assess the susceptibility and pathogenicity of antimicrobials. However, there is no suitable method for rapid and accurate laboratory detection of $N$. cyriacigeorgica. In this study, we combined PCR amplification with the CRISPR-Cas12a system to establish a novel detection platform, named CRISPR-PCR, and applied it to the detection of N. cyriacigeorgica in clinical samples. The Cas12a protein exhibited collateral cleavage activity following CRISPR RNA binding to specific targets, then indiscriminately cleaved nearby single-stranded DNA, and this was evaluated for diagnostic nucleic acid detection by measuring the fluorescence signal using a fluorescence reader. The assay takes only $2 \mathrm{~h}$, including DNA extraction for 20 min, nucleic acid pre-amplification for $70 \mathrm{~min}$, and fluorescence detection for $20 \mathrm{~min}$. The limit of detection for $N$. cyriacigeorgica was $10^{-3} \mathrm{ng}$ and the specificity was $100 \%$. Thus, the N. cyriacigeorgica CRISPR-PCR assay is a rapid and specific method for detecting N. cyriacigeorgica, and the CRISPR-PCR fluorescence detection platform has great potential for detection of other pathogens.

Keywords: Nocardia cyriacigeorgica, nucleic acid detection, CRISPR, Cas12a, CRISPR-PCR

\section{INTRODUCTION}

Nocardia bacteria are Gram-positive and partially acid-fast aerobic Actinomycetes (McTaggart et al., 2010). The genus Nocardia is ubiquitous in environments associated with decaying vegetation and deposits from animals, and members are also associated with water, soil and air (Brown-Elliott et al., 2006; Lebeaux et al., 2019). Nocardia can cause serious opportunistic infection in most organs, including traumatic infection, pulmonary disease and brain abscess (McTaggart et al., 2010; Huang et al., 2019; Lebeaux et al., 2019; Ji et al., 2020). The incidence of Nocardia infection is increased significantly in immunocompromised individuals (Minero et al., 2009; Coussement et al., 2016), including 
advanced acquired immunodeficiency syndrome (AIDS) (Castro and Espinoza, 2007), patients undergoing organ transplantation (Coussement et al., 2016) and chronic lung disease.

More than 100 species belonging to the genus Nocardia have been recognised according to the National Center for Biotechnology Information (NCBI), and more than 50 species are of clinical significance (Conville et al., 2018). Nocardia cyriacigeorgica is one of the most common agents of Nocardia infection. With an increase in immunocompromised patients and improvements in molecular identification of pathogens, the incidence of $N$. cyriacigeorgica infection is rising (Condas et al., 2013; Hashemi-Shahraki et al., 2015; Chen et al., 2017; Han et al., 2020).

Identification of Nocardia at the species level is essential to assess the susceptibility and pathogenicity of antimicrobials. Different Nocardia species have different antimicrobial susceptibility patterns, which may cause confusion during clinical treatment (Huang et al., 2019; Hamdi et al., 2020). The genome sequences of Nocardia species are highly similar (Conville et al., 2018), hence biochemical identification methods are not adequate to allow discrimination among species. The gold standard for $N$. cyriacigeorgica identification is $16 \mathrm{~S}$ rDNA sequencing, but this is time-consuming and it requires sophisticated equipment, which makes it challenging for routine laboratories (Barnaud et al., 2005; McTaggart et al., 2010). Thus, there is a growing need for a detection platform that can rapidly and accurately identify $N$. cyriacigeorgica.

The clustered regularly interspaced short palindromic repeats and CRISPR-associated protein (CRISPR-Cas) system is an adaptive immune system in bacteria and archaea that recognises and degrades foreign nucleic acid through the guidance of CRISPR RNA (crRNA) (Abudayyeh et al., 2016; Chen et al., 2018; Xiong et al., 2020). Cas proteins, including cas12a, cas12b, cas13a, cas13b and cas14, recognize and ciscleavage target DNA or RNA, and then activated Cas proteins trans-cleavage non-targeted single-stranded DNA (ssDNA) indiscriminately (Gootenberg et al., 2017; Harrington et al., 2018; Li et al., 2018; Wang et al., 2019). Therefore, nontargeted ssDNA labelled with a fluorophore and quencher produces a fluorescence signal after collateral cleavage, which can be used to detect nucleic acids (Huang et al., 2020; Xiong et al., 2020). The intensity of fluorescence signals can be measured using a fluorescence reader for specific nucleic acid detection (Zhang et al., 2021).

Recently, detection methods based on the CRISPR-Cas system have been developed, and they show great promise. The CRISPR-Cas system is commonly combined with isothermal amplification or PCR amplification to rapidly and accurately detect nucleic acids for pathogen identification (Kellner et al., 2019). Isothermal amplification, such as recombinase polymerase amplification (RPA) and loop-mediated isothermal amplification (LAMP), can be combined with Cas12 or Cas 13 for nucleic acid detection. Specific high-sensitivity enzymatic reporter (SHERLOCK) can be combined with CRISPR-Cas13a and RPA to specifically detect Zika and Dengue viruses (Gootenberg et al., 2017; Kellner et al., 2019). CRISPR-Cas12a- based detection can rapidly and accurately distinguish human papillomavirus (HPV) type 16 and type 18 using the DNA endonuclease-targeted CRISPR trans reporter (DETECTR) (Chen et al., 2018) and 1-hour low-cost multipurpose highly efficient system (HOLMES) approach (Li et al., 2018). However, isothermal amplification suffers limitations; it is prone to falsepositive results and it is expensive.

In the present study, we established a highly accurate and sensitive detection platform combining PCR amplification with CRISPR-Cas12a, termed CRISPR-PCR, to detect $N$. cyriacigeorgica (Figure 1A). Target sequences were amplified by PCR, then CRISPR-Cas12a was combined with crRNA to generate a CRISPR-Cas12a/crRNA complex (Figure 1B), which exhibited collateral cleavage of a ssDNA probe to generate a fluorescence signal. The N. cyriacigeorgica CRISPR-PCR assay can directly detect low levels of $N$. cyriacigeorgica in clinical samples, and it could help clinicians to employ appropriate antibiotic regimes in the early stages of infection.

\section{MATERIALS AND METHODS}

\section{Materials}

Primers for PCR amplification, ssDNA probe, and crRNA were synthesised by Sangon Biotech (Shanghai, China) with highperformance liquid chromatography (HPLC) purification (Table S1). LbaCas12a and 10× NEBuffer 2.1 were purchased from New England Biolabs (Beijing, China). A Wizard Genomic DNA Purification Kit was purchased from Promega (USA). DNase/ RNase-free deionised water was purchased from TIANGEN (Beijing, China). Premix Ex Taq (Probe qPCR) was purchased from TaKaRa (Dalian, China). A QuantStudio ${ }^{\text {TM }} 6$ Flix instrument $(\mathrm{X})$ was employed as a fluorescence reader.

\section{Oligonucleotide Sequence Design}

The Ncc1 gene of N. cyriacigeorgica (Accession: LR215973.1), which encodes an uncharacterised protein (Protein ID: VFA97995.1), was used for primer design. The specificity of Ncc1 was verified by BLASTn. Clustal Omega (https://www.ebi. ac.uk/Tools/msa/clustalo/) was employed for multiple sequence alignment to identify conserved regions of $N c c 1$, which served as target sequences (Figure 1C and Figure S1).

PCR primer pairs targeting conserved sequences of $\mathrm{Nccl}$ were designed using standard procedures (Figure 1C and Table S1). We screened the best primer pair and the optimum annealing temperature for subsequent experiments. The specificity of the primer pair was verified by Primer-BLAST (https://www.ncbi.nlm. nih.gov/tools/primer-blast/).

Non-targeting ssDNA fluorescent probes can be constituted by thymine polymer (poly $\mathrm{T}$ ). The ssDNA was modified by adding the fluorescent group 6-FAM at the 5' end and the quencher group BHQ1 at the 3' end (Table S1). To achieve high detection specificity, we used the highly-conserved region to design an efficient crRNA according to the CRISPR-Cas12a detection principle (Figure 1C and Table S1). 

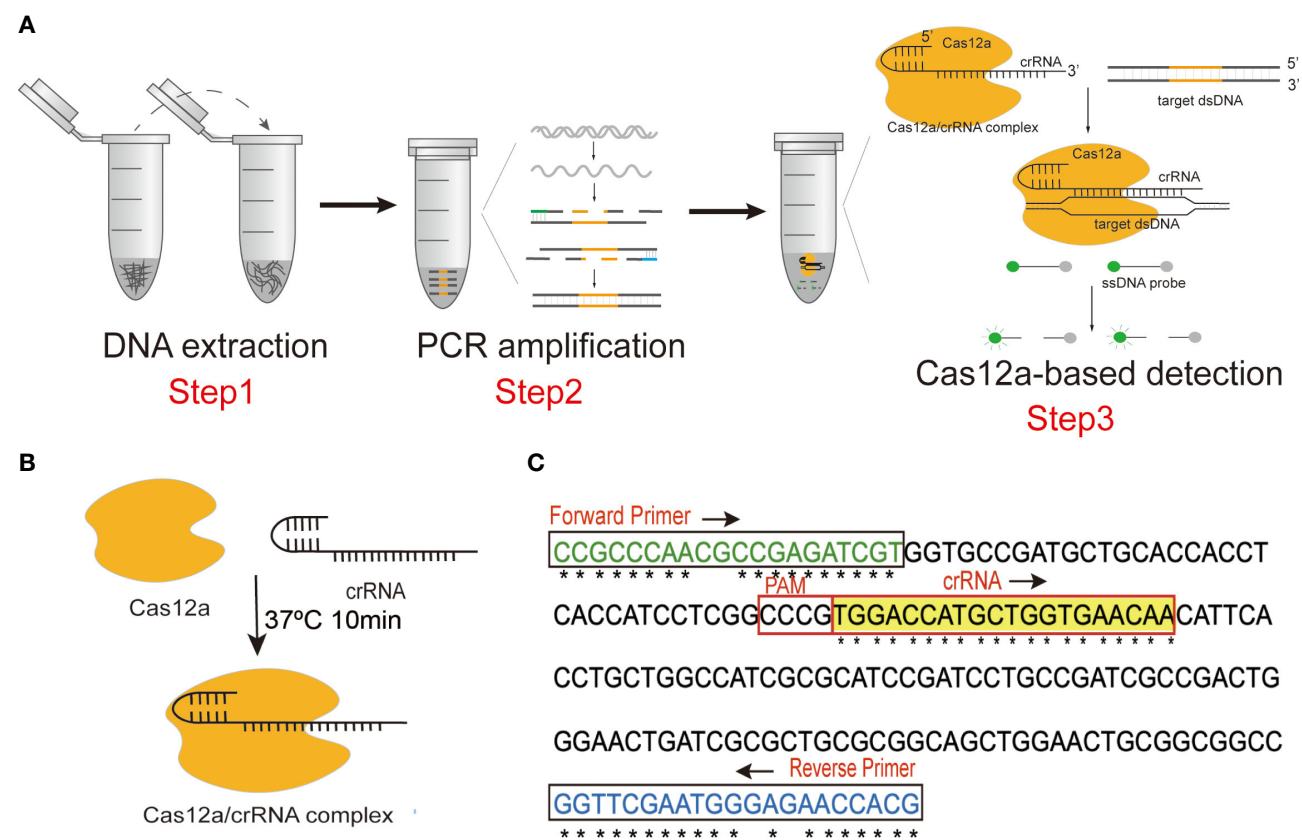

C CCGCCCAACGCCGAGATCGTGGTGCCGATGCTGCACCACCT

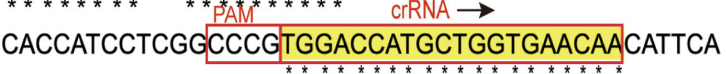
CCTGCTGGCCATCGCGCATCCGATCCTGCCGATCGCCGACTG GGAACTGATCGCGCTGCGCGGCAGCTGGAACTGCGGCGGCC $\leftarrow$ Reverse Primer GGTTCGAATGGGAGAACCACG

FIGURE 1 | (A) Schematic diagram of the N. cyriacigeorgica CRISPR-PCR assay. (B) Schematic diagram of the CRISPR-Cas12a/crRNA complex. (C) Sequences and locations of PCR primers and crRNA in this assay.

\section{Establishing the CRISPR-PCR Fluorescence Detection Assay}

A DNA fragment of the $\mathrm{Nccl}$ gene was selected as the target sequence (Figure 1C), which was amplified by PCR in a $20 \mu \mathrm{L}$ reaction containing $10 \mu \mathrm{L}$ of Premix Ex Taq (Probe qPCR), $7 \mu \mathrm{L}$ of DNase/RNase-free deionised water, $1 \mu \mathrm{L}$ of forward primer $(10 \mu \mathrm{M}), 1 \mu \mathrm{L}$ of reverse primer $(10 \mu \mathrm{M})$, and $1 \mu \mathrm{L}$ of template DNA. Thermal cycling involved an initial denaturation for $5 \mathrm{~min}$ at $95^{\circ} \mathrm{C}$ followed by 35 cycles at $95^{\circ} \mathrm{C}$ for $30 \mathrm{~s}, 65^{\circ} \mathrm{C}$ for $30 \mathrm{~s}, 72^{\circ} \mathrm{C}$ for $30 \mathrm{~s}$, and a final extension for $7 \mathrm{~min}$ at $72^{\circ} \mathrm{C}$.

The Cas12a protein was combined with crRNA to form a CRISPR-Cas12a/crRNA complex (Figure 1B) in advance to stabilise the CRISPR detection process. CRISPR-Cas12a/crRNA complexes contained $100 \mathrm{nM}$ crRNA and $75 \mathrm{nM}$ Cas12a in $1 \times$ NEBuffer 2.1, they were incubated at $37^{\circ} \mathrm{C}$ for $10 \mathrm{~min}$, and they were used immediately after preparation. The $100 \mu \mathrm{L}$ reaction volume of the CRISPR-Cas12a system included $50 \mu \mathrm{L}$ of $2 \times$ NEBuffer 2.1, $18 \mu \mathrm{L}$ of CRISPR-Cas12a/crRNA complex, $2 \mu \mathrm{L}$ of amplification product, $27.5 \mu \mathrm{L}$ of distilled water, and $2.5 \mu \mathrm{L}$ of 10 $\mu \mathrm{M}$ ssDNA probe. Fluorescence signals were detected by a fluorescence reader at $37^{\circ} \mathrm{C}$ for $20 \mathrm{~min}$. Fluorescence measurements were performed every minute to determine whether the sample contained the target sequence.

\section{Assessing the Specificity and Sensitivity of the N. cyriacigeorgica CRISPR-PCR Assay}

The specificity of the N. cyriacigeorgica CRISPR-PCR assay was verified by $60 \mathrm{~N}$. cyriacigeorgica strains (including 7 standard strains and 53 clinical strains) and 44 non- $N$. cyriacigeorgica strains (including 25 type strains of other Nocardia species and 19 strains not from the Nocardia genus; Table S2).

The genome of the N. cyriacigeorgica type strain (DSM 44484) was extracted using a Wizard Genomic DNA Purification Kit. The initial DNA concentration was $100 \mathrm{ng} / \mu \mathrm{L}$ measured by a NanoDrop-1000 instrument. To define the limit of detection (LoD) of the $N$. cyriacigeorgica CRISPR-PCR assay, we serially diluted template DNA 10 -fold intervals from $10 \mathrm{ng}$ to $10^{-4} \mathrm{ng}$, and $1 \mu \mathrm{L}$ aliquots of templates were used in CRISPR-PCR reactions. Agarose gel analysis was a control experiment for sensitivity detection.

\section{Applying the $\boldsymbol{N}$. cyriacigeorgica CRISPR- PCR Assay to Clinical Samples}

To evaluate the practical applicability of the $N$. cyriacigeorgica CRISPR-PCR assay, we assessed clinical samples. Since $N$. cyriacigeorgica is difficult to identify in routine laboratories, and because Nocardia infection is relatively rare, we spiked $N$. cyriacigeorgica bacteria liquid into clinical samples to mimic clinical situations. To assess assay sensitivity, $900 \mu \mathrm{L}$ aliquots of sputum specimens were inoculated with $100 \mu \mathrm{L}$ of a calibrated suspension of $N$. cyriacigerogica at a final concentration ranging from $10^{6}$ colony-forming units (CFU)/mL to $10^{2} \mathrm{CFU} / \mathrm{mL}$. The amplified products were separated by $2 \%$ agarose gel electrophoresis. Isolated cultures were used in a parallel experiment to assess the specificity of clinical sample detection. Briefly, 20 negative sputum specimens, which were shown to be free of N. cyriacigerogica by isolated culture, were divided into two equal parts; one part was used for preparation of positive sputum specimens, and the other served as a negative control. 
The 20 positive sputum specimens consisted of $900 \mu \mathrm{L}$ of sputum and $100 \mu \mathrm{L}$ of bacterial liquid, and equal volumes were added to $100 \mu \mathrm{L}$ of phosphate-buffered saline as negative controls. Nucleic acids extracted from the 40 sputum specimens were used as amplification templates. For this, $1 \mu \mathrm{L}$ of DNA template was added to the PCR system for amplification, and $2 \mu \mathrm{L}$ of the resulting amplicons were added to the CRISPR reaction system. Fluorescence signals were detected by a fluorescence reader at $37^{\circ} \mathrm{C}$ for $20 \mathrm{~min}$.

\section{RESULTS}

\section{Establishing the $N$. cyriacigeorgica CRISPR-PCR Assay}

We established the $N$. cyriacigeorgica CRISPR-PCR assay by combining the CRISPR-Cas12a system and PCR amplification to identify $N$. cyriacigeorgica DNA accurately and robustly, and the assay can be performed in routine laboratories. Our CRISPR-PCR assay can achieve rapid detection, and can be completed within $2 \mathrm{~h}$. The assay employs three steps, including DNA extraction, target amplification, and fluorescence signal detection (Figure 1A). We amplified part of the $\mathrm{Nccl}$ gene by PCR and added amplicons to the CRISPR-Cas12a reaction system. The CRISPR-Cas12a/crRNA complex specifically binds and cleaves target dsDNA (ciscleavage), which is complementary to crRNA, then activates collateral cleavage activity which degrades ssDNA probe (transcleavage) indiscriminately to produce fluorescence. Fluorescence signals can be observed by a fluorescence reader. Notably, the assay can potentially be used for point-of-care testing if results are analysed with a homemade UV device, by the naked eye, or by a portable fluorescence reader.

\section{Optimising the $N$. cyriacigeorgica CRISPR-PCR Assay}

Seven forward primers and five reverse primers were designed targeting the conserved region of the $\mathrm{Nccl}$ gene (Figure 1C, Table S1 and Figure S1). The mutation sites were replaced with degenerate bases $(\mathrm{N})$. In order to screen the best primer pair, a reverse primer $\mathrm{R} 4$ was used with forward primers $\mathrm{F}$ 1 to F7 (Figure S2A). The results showed that F4 yielded significant fluorescence values, and we selected F4 as the forward primer. During screening of reverse primers R1 to R5, R3 performed best (Figure S2B). Thus, the optimal primer pair was F4+R3, and this pair was used in subsequent experiments.

A PCR test at six gradient annealing temperatures (from $62^{\circ} \mathrm{C}$ to $67^{\circ} \mathrm{C}$, at $1^{\circ} \mathrm{C}$ intervals) was performed to determine the optimal reaction conditions. PCR products were detected by $2 \%$ agarose gel electrophoresis. The results indicated that there was no non-specific amplification at $65^{\circ} \mathrm{C}$, and the target band at 187 bp was bright (Figure S2C). Therefore, we considered $65^{\circ} \mathrm{C}$ to be the optimal annealing temperature.

\section{Specificity of the N. cyriacigeorgica CRISPR-PCR Assay}

The specificity of the $N$. cyriacigeorgica CRISPR-PCR assay was investigated using 104 N. cyriacigeorgica strains and non-
$N$. cyriacigeorgica strains (Table S2). The results showed that fluorescence signals were detected from $60 \mathrm{~N}$. cyriacigeorgica strains at $37^{\circ} \mathrm{C}$ for $20 \mathrm{~min}$ (Figure $\mathbf{2 A}$ ), while other strains (including 19 non-Nocardia strains and 25 other Nocardia strains) were negative (Figures S3A, B). There was a significant difference between positive and negative fluorescence values (Figure 2B). Thus, the N. cyriacigeorgica CRISPR-PCR assay is highly specific for the detection of $N$. cyriacigeorgica.

\section{Sensitivity of the $N$. cyriacigeorgica CRISPR-PCR Assay}

To evaluate the sensitivity of the $N$. cyriacigeorgica CRISPR-PCR assay, 10 -fold serial dilutions of $N$. cyriacigeorgica genomic DNA were used as templates for PCR amplification. The original concentration of DNA template measured by a NanoDrop-1000 instrument was $100 \mathrm{ng} / \mu \mathrm{L}$. We detected fluorescence values from DNA templates over a concentration range from $10 \mathrm{ng}$ to $10^{-4} \mathrm{ng}$ using a fluorescence reader. The results demonstrated that the LoD of DNA templates with detectable fluorescence was $10^{-3} \mathrm{ng}$, which was the same with agarose gel analysis (Figure 2C and Figures S4A, B). And $10^{-3} \mathrm{ng} / \mu \mathrm{l}$ was equivalent to $1.46 \times 10^{2}$ copies/ $\mu \mathrm{l}$, according to DNA copies number formula $\left(6.02 \times 10^{23}\right) \times\left(\mathrm{ng} / \mu \mathrm{l} \times 10^{-9}\right) /($ DNA length $\times 660)=$ copies $/ \mu \mathrm{l}$.

\section{Examining the Feasibility of the N. cyriacigeorgica CRISPR-PCR Assay for Clinical Samples}

We explored the sensitivity and specificity of the $N$. cyriacigeorgica CRISPR-PCR assay as a $N$. cyriacigeorgica diagnostic tool for analysis of clinical sputum samples. Negative clinical samples were spiked $N$. cyriacigeorgica to generate a series of final bacterial concentrations $\left(10^{6}, 10^{5}, 10^{4}\right.$, $10^{3}, 10^{2} \mathrm{CFU} / \mathrm{mL}$ ). The results showed that the LoD of clinical sputum samples detected by the $N$. cyriacigeorgica CRISPR-PCR assay was $10^{4} \mathrm{CFU} / \mathrm{mL}$ (Figure 3A). By comparison, the LoD for isolated cultures (Figure S5) and agarose gel electrophoresis (Figure 3B) was $10^{5} \mathrm{CFU} / \mathrm{mL}$.

Next, we tested DNA templates extracted from 20 $N$. cyriacigeorgica-positive samples and $20 \mathrm{~N}$. cyriacigeorgicanegative samples. Using the N. cyriacigeorgica CRISPR-PCR assay, fluorescence signals were detected in all 20 spiked sputum samples (Figure 3C), while no positive results were obtained from the 20 negative samples (Figure S6). These results demonstrated that the N. cyriacigeorgica CRISPR-PCR assay can sensitively and specifically detect $N$. cyriacigeorgica in clinical samples.

\section{DISCUSSION}

Currently, the gold standard for the identification of Nocardia at the species level is $16 \mathrm{~S}$ rDNA sequencing, which can achieve detailed species classification and species annotation information. The 16S rDNA sequencing technique relies on pure culture, but cultivation of $N$. cyriacigeorgica requires $2-7$ 

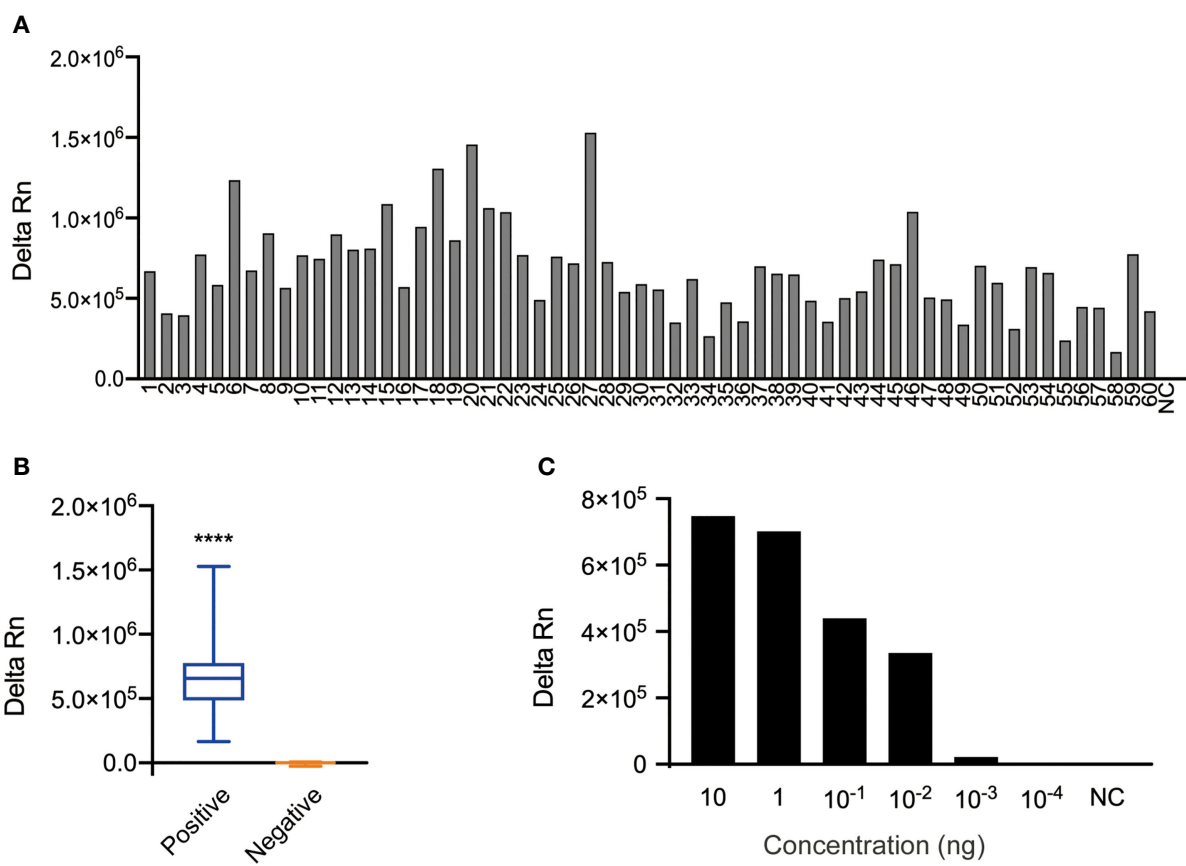

FIGURE 2 | Specificity and sensitivity of the N. cyriacigeorgica CRISPR-PCR assay. (A) Bar graph showing the fluorescence intensity of the N. cyriacigeorgica CRISPR-PCR assay at $37^{\circ} \mathrm{C}$ for $20 \mathrm{~min}$. 1-60, 60 N. cyriacigeorgica strains, including standard strains and clinical strains; NC, negative control. (B) Positive detection of $N$. cyriacigeorgica templates $(n=60)$. Templates of other strains yielded negative results $(n=45)$. The box-and-whisker plots show fluorescence values generated by the CRISPR-PCR assay at $37^{\circ} \mathrm{C}$ for 20 min. Unpaired 2-tailed $t$-tests were used to analyse differences from NC $\left({ }^{\star \star \star \star} p<0.0001\right)$. (C) Bar graph showing the fluorescence intensities for 10-fold serial dilutions of template detected by the N. cyriacigeorgica CRISPR-PCR assay at $37^{\circ} \mathrm{C}$ for $20 \mathrm{~min}$.

days, which greatly extends the time needed for identification. In addition, $16 \mathrm{~S}$ rDNA is expensive and difficult to apply in routine laboratories. Matrix-assisted laser desorption/ionisation-timeof-flight mass spectrometry (MALDI-TOF MS) is widely used in clinical practice. However, differences in the cell wall structure of Gram-positive bacteria leads to poor extraction of proteins from microbial cells, resulting in insufficient spectral information for accurate identification (Croxatto et al., 2012). Therefore, it is important to develop a novel method to rapidly and accurately detect $N$. cyriacigeorgica.

The CRISPR-Cas12a system has great potential for nucleic acid detection. Various CRISPR-Cas12a-based detection methods have been reported, including diagnostic techniques for Vibrio parahaemolyticus and SARS-CoV-2, with superior specificity and sensitivity compared with other approaches. Isothermal amplification methods are used to detect nucleic acids in combination with CRISPR-Cas techniques. Although the isothermal amplification efficiency is $10-100$ times that of PCR, it is easy to form an aerosol and cause false-positive results. Conventional PCR amplification is more stable than isothermal amplification, and has a wider range of clinical applications. Herein, we selected PCR as the nucleic acids amplification method in the first step, which has the advantages of affordability, reagent availability, and stable experimental results. Additionally, adapting conventional PCR to amplify nucleic acids greatly reduces the requirements for dedicated equipment and technical operators. The N. cyriacigeorgica CRISPR-PCR assay achieved detection using readily available equipment, which is suitable for routine clinical laboratories.

Compared with quantitative PCR and conventional PCR, the CRISPR-Cas system has the advantage of distinguishing singlebase differences. We employed the CRISPR-PCR assay to detect $N$. cyriacigeorgica. At the nucleic acid amplification stage, target sequences were exponentially amplified by PCR primers to ensure high-efficiency nucleic acid detection in the next step. After amplification, CRISPR-Cas12a recognised target sequences via crRNA for precise detection with extremely high specificity. The specificity of the N. cyriacigeorgica CRISPR-PCR assay was validated using $N$. cyriacigeorgica and other pathogenic strains. The results showed that the assay accurately detected $N$. cyriacigeorgica DNA without false-positive signals from other pathogens, confirming its specificity for N. cyriacigeorgica.

There are significant differences in the susceptibility of different Nocardia species to antibiotics. The rapid identification of Nocardia is helpful for early and rational use of antibiotics. Our CRISPR-PCR assay showed significant advantages compared with traditional detection methods based on growth characteristics, colony morphology, and biochemical reactions, which are time-consuming, laborious, and unable to achieve species-level identification. The CRISPR-PCR assay could directly detect nucleic acids extracted from clinical 


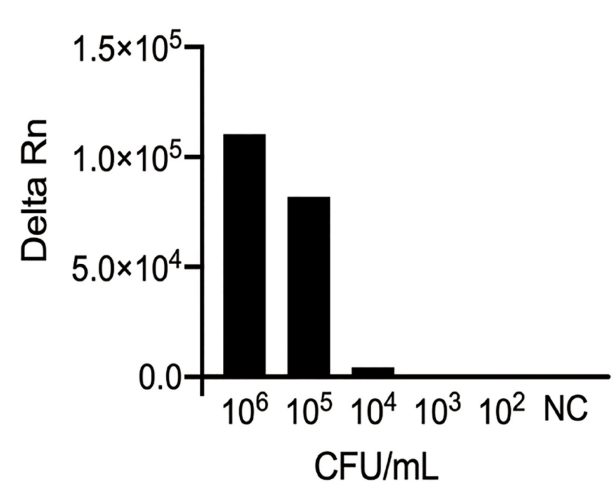

B

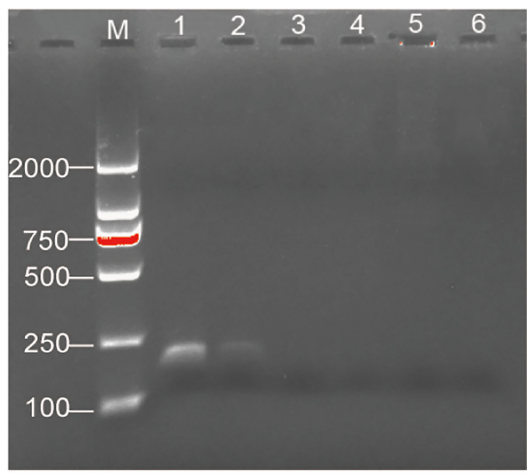

C

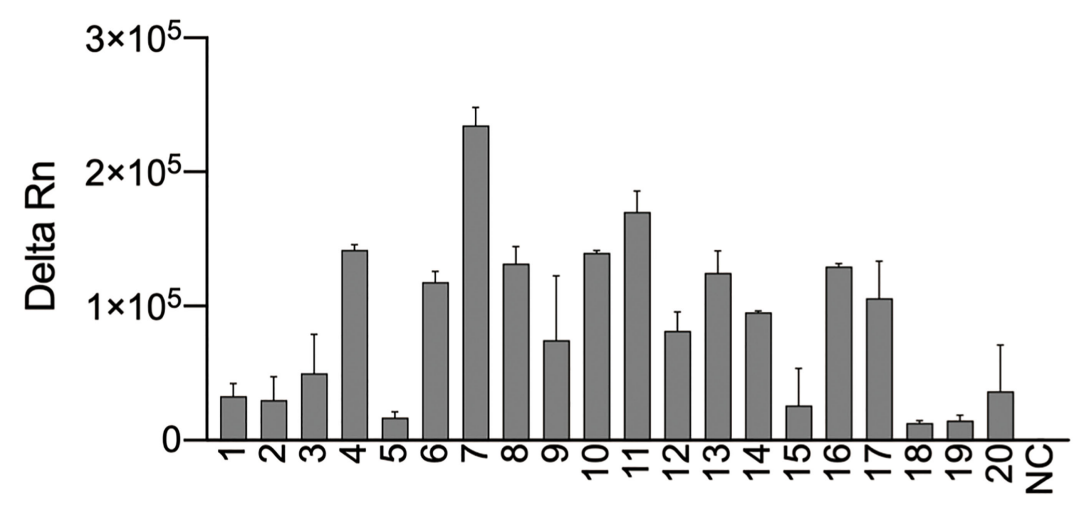

FIGURE 3 | Examination of the feasibility of the N. cyriacigeorgica CRISPR-PCR assay. (A) Fluorescence signals were measured for a series of N. cyriacigeorgica concentrations by N. cyriacigeorgica CRISPR-PCR assay at $37^{\circ} \mathrm{C}$ for $20 \mathrm{~min}$. (B) Detection of PCR products by $2 \%$ agarose gel electrophoresis. $1-5$, concentrations of DNA templates $\left(10^{6}, 10^{5}, 10^{4}, 10^{3}, 10^{2} \mathrm{CFU} / \mathrm{mL}\right) ; 6$, negative control (NC). (C) Fluorescence values for $\mathrm{N}$. cyriacigeorgica detection from spiked sputum specimens measured by N. cyriacigeorgica CRISPR-PCR assay at $37^{\circ} \mathrm{C} .1-20$, positive samples; NC, negative control.

samples to rapidly and accurately identify N. cyriacigeorgica, and the entire process can be completed within $2 \mathrm{~h}$. The LoD of the CRISPR-PCR assay was $10^{4} \mathrm{CFU} / \mathrm{mL}$ for clinical sputum specimens, significantly better than $10^{5} \mathrm{CFU} / \mathrm{mL}$ for isolated culture. The results showed that CRISPR-PCR enables rapid and sensitive detection of $N$. cyriacigeorgica and avoids the subjectivity of visual observation, hence the assay may be more suitable for the detection of $N$. cyriacigeorgica infection in clinical samples.

Our previous work CRISPR-CPA and CRISPR-PCR were CRISPR-Cas12a-based detection combined with PCR preamplification. However, the detection strategies of the two methods were different. In CRISPR-CPA system, a Protospacer adjacent motif site (PAM) sequences TTTA and a protective base were added at the 5 ' end of forward primer, and the crRNA was designed at the downstream of the PAM site (Qiu et al., 2021). Nevertheless, CRISPR-CPA may not be suitable to detect $N$. cyriacigeorgica, because the species-specific gene used in this study was not conserved enough, which was due to the high intraspecific variability of $N$. cyriacigeorgica. our CRISPR-PCR assay, TTTA sequence was not used as a recognition site for Cas12a to initiate CRISPR-based nucleic acid detection. We used non-canonical C-containing sequences as PAM sites to guide the Cas12a/crRNA complex to bind to the target sequence. According to previous studies, LbCas12a possessed extensive PAM sites and non-canonical Ccontaining sequences could be suboptimal PAM sites (Tran et al., 2021; Dronina et al., 2022). The application of Cas12a to nucleic acid detection relies on trans-cleavage activity. The collateral cleavage activity of the CRISPR-Cas12a system is independent of target sequences cleavage and trans-cleavage activity is triggered upon binding of the Cas $12 \mathrm{a} / \mathrm{crRNA}$ complex to target sequences (Chen et al., 2018). Therefore, in this study, our system is sufficient to trigger trans-cleavage activity to detect the target.

The N. cyriacigeorgica CRISPR-PCR assay also has several limitations. One limitation of this study is that we did not use $N$. cyriacigeorgica clinical specimens to evaluate our CRISPR-PCR fluorescence detection platform. Clinical samples such as sputum, blood and bronchoalveolar lavage fluid need to be tested in future. Another limitation of this method is that only a single species of Nocardia can be identified, and multiple Nocardia strains cannot be distinguished at the same time, and this requires further exploration. 


\section{CONCLUSION}

In this study, we established a sensitive and specific detection method that combined the CRISPR-Cas12a system with PCR amplification for detection of $N$. cyriacigeorgica DNA. The intensity of the fluorescence signal can be measured by a fluorescence reader, and the whole process is completed within $2 \mathrm{~h}$. The assay is suitable for applying to conventional microorganism laboratories, and it can reliably detect $N$. cyriacigeorgica using ordinary equipment.

\section{DATA AVAILABILITY STATEMENT}

The original contributions presented in the study are included in the article/Supplementary Material. Further inquiries can be directed to the corresponding author.

\section{ETHICS STATEMENT1}

The manuscript contains experiments using sputum specimens from human and the study was approved by the Research Ethics Committee of National Institute for Communicable Disease

\section{REFERENCES}

Abudayyeh O. O., Gootenberg J. S., Konermann S., Joung J., Slaymaker I. M., Cox D. B., et al. (2016). C2c2 Is a Single-Component Programmable RNA-Guided RNA-Targeting CRISPR Effector. Science 353 (6299), aaf5573. doi: 10.1126/ science.aaf5573

Barnaud G., Deschamps C., Manceron V., Mortier E., Laurent F., Bert F., et al. (2005). Brain Abscess Caused by Nocardia Cyriacigeorgica in a Patient With Human Immunodeficiency Virus Infection. J. Clin. Microbiol. 43 (9), 48954897. doi: 10.1128/JCM.43.9.4895-4897.2005

Brown-Elliott B. A., Brown J. M., Conville P. S., Wallace R. J.Jr. (2006). Clinical and Laboratory Features of the Nocardia Spp. Based on Current Molecular Taxonomy. Clin. Microbiol. Rev. 19 (2), 259-282. doi: 10.1128/CMR.19.2.259282.2006

Castro J. G., Espinoza L. (2007). Nocardia Species Infections in a Large County Hospital in Miami: 6 Years Experience. J. Infect. 54 (4), 358-361. doi: 10.1016/ j.jinf.2006.08.003

Chen W., Liu Y., Zhang L., Gu X., Liu G., Shahid M., et al. (2017). Nocardia Cyriacigeogica From Bovine Mastitis Induced In Vitro Apoptosis of Bovine Mammary Epithelial Cells via Activation of Mitochondrial-Caspase Pathway. Front. Cell Infect. Microbiol. 7, 194. doi: 10.3389/fcimb.2017.00194

Chen J. S., Ma E., Harrington L. B., Da Costa M., Tian X., Palefsky J. M., et al. (2018). CRISPR-Cas12a Target Binding Unleashes Indiscriminate SingleStranded DNase Activity. Science. 360 (6387), 436-439. doi: 10.1126/ science.aar6245

Condas L. A., Ribeiro M. G., Yazawa K., de Vargas A. P., Salerno T., Giuffrida R., et al. (2013). Molecular Identification and Antimicrobial Susceptibility of Nocardia Spp. Isolated From Bovine Mastitis in Brazil. Vet. Microbiol. 167 (3-4), 708-712. doi: 10.1016/j.vetmic.2013.08.019

Conville P. S., Brown-Elliott B. A., Smith T., Zelazny A. M. (2018). The Complexities of Nocardia Taxonomy and Identification. J. Clin. Microbiol. 56 (1), e01419-17. doi: 10.1128/JCM.01419-17

Coussement J., Lebeaux D., van Delden C., Guillot H., Freund R., Marbus S., et al. (2016). Nocardia Infection in Solid Organ Transplant Recipients: A Multicenter European Case-Control Study. Clin. Infect. Dis. 63 (3), 338-345. doi: $10.1093 / \mathrm{cid} / \operatorname{ciw} 241$
Control and Prevention, Chinese Center for Disease Control and Prevention (No. ICDC-2021004).

\section{AUTHOR CONTRIBUTIONS}

$\mathrm{XL}, \mathrm{XQ}$, and SX conceived and designed the experiments. XL and $\mathrm{XQ}$ wrote the manuscript and performed the experiments. YC, LH, YK, YY, SC, and FL analysed the data and contributed reagents. ZL provided financial and administrative support. All authors contributed to the article and approved the submitted version.

\section{FUNDING}

This work was supported by the Biosafety Key Special Project (2019YFC1200700, 2019YFC1200601) and the National Natural Science Foundation of China (no. 82073624).

\section{SUPPLEMENTARY MATERIAL}

The Supplementary Material for this article can be found online at: https://www.frontiersin.org/articles/10.3389/fcimb.2022. 835213/full\#supplementary-material

Croxatto A., Prod'hom G., Greub G. (2012). Applications of MALDI-TOF Mass Spectrometry in Clinical Diagnostic Microbiology. FEMS Microbiol. Rev. 36 (2), 380-407. doi: 10.1111/j.1574-6976.2011.00298.x

Dronina J., Samukaite-Bubniene U., Ramanavicius A. (2022). Towards Application of CRISPR-Cas12a in the Design of Modern Viral DNA Detection Tools (Review). J. Nanobiotechnology 20 (1), 41. doi: 10.1186/ s12951-022-01246-7

Gootenberg J. S., Abudayyeh O. O., Lee J. W., Essletzbichler P., Dy A. J., Joung J. et al. (2017). Nucleic Acid Detection With CRISPR-Cas13a/C2c2. Science 356 (6336), 438-442. doi: 10.1126/science.aam9321

Hamdi A. M., Fida M., Deml S. M., Abu Saleh O. M., Wengenack N. L. (2020). Retrospective Analysis of Antimicrobial Susceptibility Profiles of Nocardia Species From a Tertiary Hospital and Reference Laboratory, 2011 to 2017. Antimicrob. Agents Chemother. 64 (3), e01868-19. doi: 10.1128/AAC.01868-19

Han L., Ji X., Xu S., Fan S., Wang C., Wei K., et al. (2020). Microbiological Profile of Distinct Virulence of Nocardia Cyriacigeorgica Strains In Vivo and In Vitro. Microb. Pathog. 142, 104042. doi: 10.1016/j.micpath.2020.104042

Harrington L. B., Burstein D., Chen J. S., Paez-Espino D., Ma E., Witte I. P., et al. (2018). Programmed DNA Destruction by Miniature CRISPR-Cas14 Enzymes. Science. 362 (6416), 839-842. doi: 10.1126/science.aav4294

Hashemi-Shahraki A., Heidarieh P., Bostanabad S. Z., Hashemzadeh M., Feizabadi M. M., Schraufnagel D., et al. (2015). Genetic Diversity and Antimicrobial Susceptibility of Nocardia Species Among Patients With Nocardiosis. Sci. Rep. 5, 17862. doi: $10.1038 /$ srep 17862

Huang L., Chen X., Xu H., Sun L., Li C., Guo W., et al. (2019). Clinical Features, Identification, Antimicrobial Resistance Patterns of Nocardia Species in China: 2009-2017. Diagn. Microbiol. Infect. Dis. 94 (2), 165-172. doi: 10.1016/ j.diagmicrobio.2018.12.007

Huang Z., Tian D., Liu Y., Lin Z., Lyon C. J., Lai W., et al. (2020). Ultra-Sensitive and High-Throughput CRISPR-P Owered COVID-19 Diagnosis. Biosens Bioelectron. 164, 112316. doi: 10.1016/j.bios.2020.112316

Ji X., Zhang X., Sun L., Hou X., Song H., Han L., et al. (2020). The HeparinBinding Hemagglutinin of Nocardia Cyriacigeorgica GUH-2 Stimulates Inflammatory Cytokine Secretion Through Activation of Nuclear Factor $\mathrm{\kappa b}$ and Mitogen-Activated Protein Kinase Pathways via TLR4. Front. Cell Infect. Microbiol. 10, 3. doi: 10.3389/fcimb.2020.00003 
Kellner M. J., Koob J. G., Gootenberg J. S., Abudayyeh O. O., Zhang F. (2019). SHERLOCK: Nucleic Acid Detection With CRISPR Nucleases. Nat. Protoc. 14 (10), 2986-3012. doi: 10.1038/s41596-019-0210-2

Lebeaux D., Bergeron E., Berthet J., Djadi-Prat J., Mouniée D., Boiron P., et al. (2019). Antibiotic Susceptibility Testing and Species Identification of Nocardia Isolates: A Retrospective Analysis of Data From a French Expert Laboratory, 2010-2015. Clin. Microbiol. Infect. 25 (4), 489-495. doi: 10.1016/ j.cmi.2018.06.013

Li S. Y., Cheng Q. X., Liu J. K., Nie X. Q., Zhao G. P., Wang J. (2018). CRISPRCas12a has Both Cis- and Trans-Cleavage Activities on Single-Stranded DNA. Cell Res. 28 (4), 491-493. doi: 10.1038/s41422-018-0022-X

Li S. Y., Cheng Q. X., Wang J. M., Li X. Y., Zhang Z. L., Gao S., et al. (2018). CRISPR-Cas12a-Assisted Nucleic Acid Detection. Cell Discov. 4, 20. doi: 10.1038/s41421-018-0028-Z

McTaggart L. R., Richardson S. E., Witkowska M., Zhang S. X. (2010). Phylogeny and Identification of Nocardia Species on the Basis of Multilocus Sequence Analysis. J. Clin. Microbiol. 48 (12), 4525-4533. doi: 10.1128/JCM.00883-10

Minero M. V., Marín M., Cercenado E., Rabadán P. M., Bouza E., Muñoz P. (2009). Nocardiosis at the Turn of the Century. Med. (Baltimore) 88 (4), 250261. doi: 10.1097/MD.0b013e3181afa1c8

Qiu X., Xu S., Liu X., Han L., Zhao B., Che Y., et al. (2021). A CRISPR-Based Nucleic Acid Detection Platform (CRISPR-CPA): Application for Detection of Nocardia Farcinica. J. Appl. Microbiol. 00, 1-9. doi: 10.1111/jam.15424

Tran M. H., Park H., Nobles C. L., Karunadharma P., Pan L., Zhong G., et al. (2021). A More Efficient CRISPR-Cas12a Variant Derived From Lachnospiraceae Bacterium MA2020. Mol. Ther. Nucleic Acids 24, 40-53. doi: 10.1016/j.omtn.2021.02.012
Wang B., Wang R., Wang D., Wu J., Li J., Wang J., et al. (2019). Cas12aVDet: A CRISPR/Cas12a-Based Platform for Rapid and Visual Nucleic Acid Detection. Anal. Chem. 91 (19), 12156-12161. doi: 10.1021/acs.analchem.9b01526

Xiong D., Dai W., Gong J., Li G., Liu N., Wu W., et al. (2020). Rapid Detection of SARS-CoV-2 With CRISPR-Cas12a. PloS Biol. 18 (12), e3000978. doi: 10.1371/ journal.pbio.3000978

Zhang W. S., Pan J., Li F., Zhu M., Xu M., Zhu H., et al. (2021). Reverse Transcription Recombinase Polymerase Amplification Coupled With CRISPRCas12a for Facile and Highly Sensitive Colorimetric SARS-CoV-2 Detection. Anal. Chem. 93 (8), 4126-4133. doi: 10.1021/acs.analchem.1c00013

Conflict of Interest: The authors declare that the research was conducted in the absence of any commercial or financial relationships that could be construed as a potential conflict of interest.

Publisher's Note: All claims expressed in this article are solely those of the authors and do not necessarily represent those of their affiliated organizations, or those of the publisher, the editors and the reviewers. Any product that may be evaluated in this article, or claim that may be made by its manufacturer, is not guaranteed or endorsed by the publisher.

Copyright (C) 2022 Liu, Qiu, Xu, Che, Han, Kang, Yue, Chen, Li and Li. This is an open-access article distributed under the terms of the Creative Commons Attribution License (CC BY). The use, distribution or reproduction in other forums is permitted, provided the original author(s) and the copyright owner(s) are credited and that the original publication in this journal is cited, in accordance with accepted academic practice. No use, distribution or reproduction is permitted which does not comply with these terms. 